\title{
Inversion of the intensity-magnetic field relationship in solar active regions
}

\author{
A. Fludra ${ }^{1}$ and J. Ireland ${ }^{2}$ \\ 1 Space Science \& Technology Department, Rutherford Appleton Laboratory, Chilton, Didcot, Oxfordshire, OX11 0QX, UK \\ ${ }^{2}$ L-3 Communications/EER Systems Inc., NASA Goddard Space Flight Center, Greenbelt, MD 20771, USA
}

Received 6 June 2002 / Accepted 4 November 2002

\begin{abstract}
We discuss the relationship between the EUV spectral line intensities and the photospheric magnetic flux in solar active regions. Since the histograms of the magnetic flux density in active region plages can be approximated by an exponential function, the equation describing how the observed total line intensity integrated over an active region area arises from the magnetic field, can be approximated by a Laplace integral. We use this property to solve an inverse problem and derive a function relating the line intensity from individual loops to the photospheric magnetic flux density at their footpoints. We propose a simple model in which the intensity of a coronal line Fe XVI $360.8 \AA$ in an individual coronal loop is proportional to the footpoint magnetic flux density to the power of $\delta$ and explore how well the value of $\delta$ is constrained by the observations. Using EUV spectra from the Coronal Diagnostic Spectrometer (CDS) on SOHO and magnetograms from SOHO Michelson Doppler Imager for 26 active regions without sunspots, we find that the value of $\delta$ depends on the magnetic flux density threshold used to define active region magnetic area. When even the weakest fields are included, we obtain $\delta=1.3$, where $1.0<\delta<1.6$ with $90 \%$ confidence. This result can be used to provide constraints on coronal heating models.
\end{abstract}

Key words. Sun: magnetic field - Sun: corona - Sun: UV radiation

\section{Introduction}

Despite the abundance of theoretical models of coronal heating, the mechanism that heats the solar corona has not yet been identified. Observational constraints on the heating mechanism can be obtained from the comparison of the spectral line emission and the magnetic flux in active regions. The intensity of the EUV and X-ray emission in coronal loops depends on the temperature and density distributions along the loop which are determined by the heating rate which, in turn, is predicted to depend on the magnetic field strength, loop length and other parameters (see Mandrini et al. 2000 for a review of coronal heating models). Thus, if well-defined relationships exist between the observed spectral line intensities and the magnetic flux integrated over the active region area, they carry information that can help one identify which models are plausible. The goal of this paper is to present a new method for establishing such a relationship, illustrate its application using a coronal EUV line of Fe XVI $360.8 \AA$ integrated over the active region area, and explore how well this global relationship constrains the intensitymagnetic flux dependence for individual loops.

Past research has shown that the total intensity of EUV spectral lines or a broad-band X-ray flux summed over an entire

Send offprint requests to: A. Fludra,

e-mail: A.Fludra@rl.ac.uk active region are related to the global magnetic quantities. For example, Gurman et al. (1974) studied OSO-6 data and found a proportionality of the $\mathrm{Mg}$ X $624.9 \AA$ line intensity to the magnetic flux density. Schrijver (1987) used Skylab data which included a chromospheric C II line, a coronal Mg X 624.9 Å line and soft X-ray emission and found power laws relating integrated intensities to the total magnetic flux $\Phi$, with the power index dependent on where the spectral line is emitted (chromosphere, transition region or corona). The $I_{\mathrm{x}-\text { ray }} \propto \Phi_{t}^{1.2}$ power law (Schrijver 1987), even though obtained from a small sample of 17 active regions was later confirmed by Fisher et al. (1998) from a study of 330 active regions observed by the Yohkoh Soft X-ray Telescope. Fisher et al. (1998) explored correlations between the total X-ray luminosity and three quantities: the total magnetic area, the total unsigned magnetic flux, and the total $B^{2}$, concluding that the X-ray luminosity correlates better with the total unsigned flux than with the total of $B^{2}$.

In this paper, we extend the previous analyses by seeking the underlying causes from which these power laws arise. We calculate spatially integrated EUV line intensities, but treat the magnetic field in a more detailed way. Instead of arbitrarily selecting one or two integrated magnetic quantities, we use histograms of the magnetic flux density to derive the dependence of the line intensities in individual loops on the magnetic flux density at their footpoints. We adopt a simple model of this 
dependence and explore how this model is constrained by the total line intensities integrated over the active region area.

The solution to the coronal heating problem can be approached in two stages: first, one can derive the observed dependence of the spectral line intensities on the magnetic field; second, the dependence of line intensities on the heating rate can be established using loop models. In this paper we address the first step, assessing how precisely the relationships between the intensities in individual loops and the magnetic flux at their footpoints can be established from the spatially integrated intensities and magnetic flux density histograms.

Ideally, one would wish to find a relationship between the spectral emission in individual coronal loops and the coronal magnetic field strength. However, given the difficulties with measuring coronal magnetic fields, and the common availability of photospheric field measurements from ground-based and space magnetographs, we are seeking relationships between the spectral emission and the photospheric magnetic flux density at loop footpoints. The most accurate approach is to make a direct spatial comparison between the EUV images and magnetograms, by identifying individual coronal loops, tracing their shape down to the photospheric levels and identifying their footpoints in the magnetograms. This approach was attempted by Aschwanden et al. (1999) using SOHO Extreme-Ultraviolet Imaging Telescope (EIT) data for selected loops in selected active regions. It is clear, however, that the complexity of the active region structure and the precision required for this task allow it to be performed only for selected coronal loops. For complex active region structures, particularly when observed with a moderate spatial resolution $\left(\geq 2^{\prime \prime}\right)$, alternative ways must be sought to infer the $I-B$ relationship in individual loops from a statistical analysis of a large number of active regions. For example, one could simulate the surface magnetic field and compute the corresponding coronal field through potential field extrapolation (Schrijver \& Aschwanden 2002).

In contrast to previous analyses which have either concentrated on global averages or measured individual loops, our approach goes forward by making an assumption on the ensemble properties of loops in an active region. The solution looks at the distribution of magnetic field elements in an active region, coupled with a model assumption on the individual intensity-field relationship. This model establishes a relationship between the total EUV line intensity in an individual coronal loop, and the photospheric magnetic flux density at its footpoints, and thus extends previous analyses which discussed only spatially-integrated quantities.

The parameters of our model are found by solving an inverse problem. While a general numerical solution is possible, we recognise for the first time that the mathematical form of the relationship between the spectral line emission integrated over the active region area and the magnetic flux density distribution in the active region plage is a Laplace transform. The solution is therefore based on an inverse Laplace transform which offers additional insights into the nature of this problem.

As an illustration, we use calibrated EUV spectra from the Coronal Diagnostic Spectrometer (CDS; Harrison et al. 1995) on SOHO which offer an opportunity to study the spectral line Fe XVI $360.8 \AA$, not previously used in this type of analysis.
The peak emissivity of the Fe XVI $360.8 \AA$ line is at 2 million degrees. Thus, this line provides a very good diagnostic of the active region temperatures, in contrast to the one-million degree line of $\mathrm{Mg} \mathrm{X}$, available in the previous Skylab study, which was shown to be a less useful diagnostic (Fludra \& Ireland 2002). The present study examines 26 active regions without sunspots.

The model is formulated in Sect. 2, application to EUV spectra is shown in Sect. 3, and discussion is in Sect. 4.

\section{Laplace integral in active regions}

Here we are concerned with the active region magnetic plage which is the source of magnetic loops that contain hot $\left(T_{\mathrm{e}}>\right.$ $1.6 \times 10^{6} \mathrm{~K}$ ) coronal plasma. While it has now been accepted that the true photospheric magnetic field strength is at least 1-2 kG both in the quiet sun network (Stenflo 1973) and in the active region plage (Rabin 1992), magnetographs measure magnetic flux density, $\psi=<f B>$, averaged over the pixel area, where $f$ is the magnetic flux tube filling factor, and $B$ is the field strength. Such spatially averaged values of $|\psi|$ are in the range up to $600 \mathrm{Mx} \mathrm{cm}^{-2}$ outside sunspots. Thereafter, we will often refer to the unsigned magnetic flux density, denoted as $\phi=|\psi|$.

Because the magnetic pressure becomes increasingly larger than the plasma pressure with increasing height above the photosphere, the magnetic flux tubes expand with height and merge together in the low chromosphere to fill all space at heights above several hundred kilometers (the filling factor $f \approx 1$ in and above the low chromosphere). Therefore, the photospheric magnetic flux density is a good approximation of the chromospheric field strength and can be used as a measure of field strength at loop footpoints at the chromospheric level (R. Moore 2001, private communication).

Let $H(\phi)$ denote the distribution of the unsigned magnetic flux density in an active region plage (Fig. 1). We assume that a majority of magnetic field lines have both footpoints in the same active region, and that a spectral line intensity emitted from a loop depends on the magnetic flux at its footpoints. In other words, each magnetic pixel, with the unsigned magnetic flux density $\phi$, is located at a footpoint of some coronal loop with length $L$ and contributes intensity $I(\phi, L)$ to the emission from that loop, where the function $I(\phi, L)$ may be different for different spectral lines. The length $L$ for a particular coronal loop is determined by the geometry of all magnetic field sources in the active region and may be different for each loop. The total line intensity emitted from a coronal loop is thus equal to the sum of contributions arising from all pixels located at its footpoints, $I_{\text {loop }}=\Sigma I_{j}(\phi, L)$. In the simplest case of a loop joining two pixels of identical unsigned magnetic flux density $\phi$, $I_{\text {loop }}=2 I(\phi, L)$.

Thus, the total spectral line intensity from an entire active region is defined by summing intensities from all loops:

$I_{\mathrm{tot}}=\int_{a}^{b} I(\phi, L) H(\phi) \mathrm{d} \phi$ 
where the integration limits $a$ and $b$ are discussed in Sect. 3 . To simplify the notation, we set $I(\phi, L) \equiv I(\phi)$ in subsequent derivations. We address the effect of $L$ in Sect. 3.2.

Equation (1) is the so-called Fredholm equation of the first kind. Deriving the function $I(\phi)$ based on measured values $I_{\text {tot }, j}$ and known kernel functions $H_{j}(\phi)(j=1, \ldots, N)$ is a typical inverse problem. It is encountered, for example, when calculating the differential emission measure in the solar corona (e.g., Fludra \& Sylwester 1986).

In a general way, the function $I(\phi)$ can be found by the regularisation method, by minimising the following goodness-of-fit criterion

$C=\chi^{2}+\Lambda * R$

where

$\chi^{2}=\Sigma_{j=1}^{N} \frac{\left(I_{\mathrm{obs}, j}-I_{\mathrm{pred}, j}\right)}{\sigma_{j}^{2}}$.

Here the summation is carried out over $N$ active regions $(j=$ $1, \ldots, N), I_{\mathrm{obs}, j}$ are observed, spatially integrated line intensities in each region, $I_{\mathrm{pred}, j}$ are intensities predicted from Eq. (1), and $\sigma_{j}$ are measurement errors assigned to the observed intensities $I_{\mathrm{obs}, j}$.

The parameter $\Lambda$ is called the Lagrange multiplier, and $R$ is a function of $I(\phi)$ that ensures a smooth form of $I(\phi)$, for example, an integral of the square of the first or second derivative (e.g., Fludra \& Sylwester 1986).

This approach is recommended in inverse problems which lack a parametric description of the desired function $I(\phi)$ and particularly when the number of derived values of $I(\phi)$ exceeds the number of equations $N$. In this paper, however, we simplify the problem by defining $I(\phi)$ as a function of only two parameters which can then be determined by minimisation of $\chi^{2}$ alone.

Examination of histograms of the magnetic flux density distributions in active region plages, created with a step $\mathrm{d} \phi=$ $10 \mathrm{G}$, reveals that majority of them can be approximated by an exponential function of $\phi$ for $\phi>50 \mathrm{G}$, up to approximately $500 \mathrm{G}$. For $\phi<50 \mathrm{G}$, the distribution of $\phi$ has a different slope due to the quiet sun magnetic field present in the active region field of view. Figure 1 shows an example of a magnetic field histogram obtained from the SOHO Michelson Doppler Imager (MDI; Scherrer et al. 1995), and an exponential fit. Thus, we set:

$$
H(\phi)=H_{0} \exp (-\beta \phi)
$$

where $\beta$ is the decay constant and $H_{0}$ is also constant for a given active region. A detailed model of a distribution of magnetic flux concentrations in an active region plage is presented by Schrijver et al. (1997).

Using the form of $H(\phi)$ given by Eq. (4), dividing both sides of Eq. (1) by $H_{0}$ and setting $a=0, b=\infty$, we obtain in each active region:

$I_{\infty} / H_{0}=\int_{0}^{\infty} I(\phi) \exp (-\beta \phi) \mathrm{d} \phi$.

Note that the intensity on the left-hand side of Eq. (5) is denoted as $I_{\infty}$ which is not necessarily equal to $I_{\text {tot }}$ from Eq. (1) due

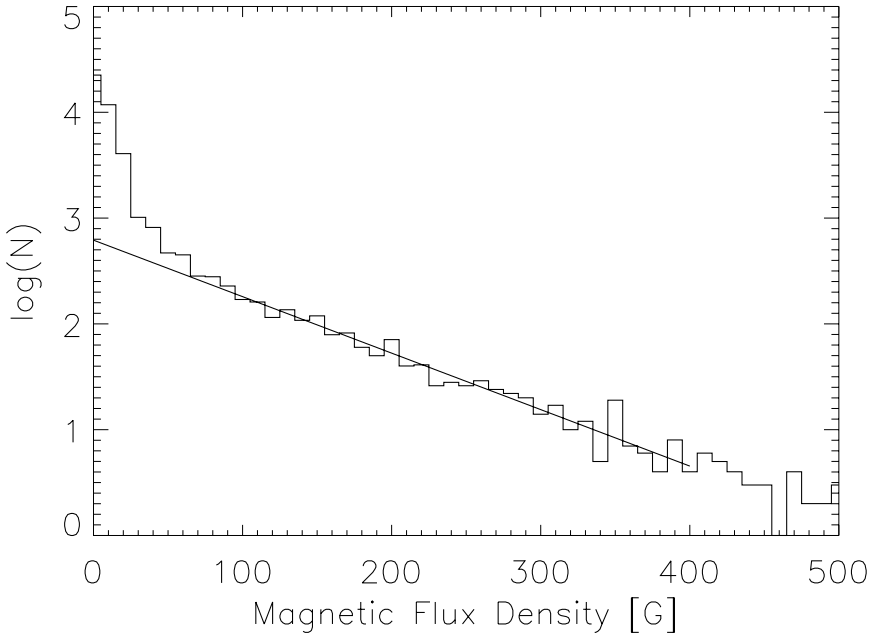

Fig. 1. Histogram of the magnetic flux density in an active region NOAA 8004 on 1996 December 17 at 01:36 UT. The continuous line is an exponential fit in the range $50-400 \mathrm{G}$.

to different integral limits. The right-hand side of Eq. (5) is a Laplace transform. The Laplace transform of a function $f(t)$ is given as: $F(s)=\int_{0}^{\infty} f(t) \exp (-s t) \mathrm{d} t$, where $0 \leq s<\infty$.

Equation 5 has now become a special type of inverse problem. The unknown contribution $I(\phi)$ to a coronal loop intensity, arising due to photospheric magnetic flux density $\phi$ in a pixel at the loop footpoint, can be obtained by performing an inverse Laplace transform, when $I_{\infty}$ is known. Various approaches to the inversion of the Laplace integral equation are discussed in Craig \& Brown (1986). Generally, if we have no prior knowledge about the shape of $I(\phi)$, the inversion of the Laplace transform is an ill-conditioned problem. However, for this application, additional information in the form of a chain of arguments below allows us to choose a particular form of $I(\phi)$.

The first clue comes from the definition of the line intensity. The line intensity $I(z)$ at a distance $(z, z+\mathrm{d} z)$ along a magnetic loop is:

$I(z)=A_{\mathrm{el}} S_{\mathrm{cr}} N_{\mathrm{e}}^{2}(z) G\left(T_{\mathrm{e}}(z)\right) \mathrm{d} z$

where $A_{\mathrm{el}}$ is the element's abundance relative to hydrogen, $S_{\text {cr }}$ is loop cross-section and $G(T)$ is the theoretical line emissivity. The electron density is expressed as $N_{\mathrm{e}}(z)=P /\left(2 k_{\mathrm{B}} T_{\mathrm{e}}(z)\right)$, where $P$ is pressure, $T_{\mathrm{e}}$ is electron temperature and $k_{\mathrm{B}}$ is Boltzmann's constant. For an approximately constant pressure along the loop, the total line intensity $I_{\text {loop }}$ emitted from the whole loop is

$I_{\text {loop }}=\frac{A_{\mathrm{el}} S_{\mathrm{cr}}}{4 k_{\mathrm{B}}^{2}} P^{2} \int_{0}^{2 L} G\left(T_{\mathrm{e}}(z)\right) / T_{\mathrm{e}}^{2}(z) \mathrm{d} z$

where the integration takes place from one loop footpoint $(z=0)$ to the other footpoint $(z=2 L)$, and $L$ is the loop halflength. The line emissivity $G(T)$ can be calculated using standard methods of atomic physics, e.g., ADAS software package (Summers 1993) or Chianti package (Dere et al. 1997).

Making a further assumption that the hot active region loops are in a static equilibrium (shown to be true for X-ray loops by Porter \& Klimchuk 1995), it is found 
(Fludra \& Ireland 2002) that the dependence of the integral on the right-hand side of Eq. (7) on pressure can be approximated as being proportional to $P^{c}$, with values of the power index $c$ depending on the loop apex temperature.

Furthermore, for a static loop, pressure is related to the average heating rate $E_{H}$ by a scaling law $E_{H}=C_{1} P^{7 / 6} L^{-5 / 6}$, where $L$ is the loop half-length and $C_{1}=1.1 \times 10^{5}$ in cgs units (Rosner et al. 1978; Martens et al. 2000). In turn, the heating rate predicted from coronal heating models (Mandrini et al. 2000), depends on the magnetic field strength $B$ through another power law, $E_{H} \propto B^{\alpha} L^{\lambda}$, where the power index $\alpha$ depends on a particular model and can range from $-3 / 2$ to $+5 / 2$ and $\lambda$ also depends on the heating model.

Combining the above arguments, we are seeking solutions to Eq. (5) of the form

$I(\phi)=Q_{0} \phi^{\delta}$

where $\delta>0$. Note that this form for $I(\phi)$ tacitly assumes that $Q_{0}$ is the same for all loops in all active regions. The effect of relaxing this assumption (by including an active region length scale dependence) is explored in Sect. 3.2.

The form of $I(\phi)$ given by Eq. (8) significantly simplifies the inversion of Eq. (5) because the Laplace transform of a function $t^{r}, r>-1$, is $\Gamma(r+1) / s^{r+1}$, where $\Gamma$ is a Gamma function. Thus, the Laplace transform of a function $\phi^{\delta}$ is $\Gamma(\delta+1) / s^{\delta+1}$ for $\delta>-1$, and Eq. (5) takes the form

$$
I_{\infty} / H_{0}=\frac{Q_{0} \Gamma(\delta+1)}{\beta^{\delta+1}} .
$$

Therefore, we only need to find two parameters, $Q_{0}$ and $\delta$. We do this by minimising a goodness-of-fit criterion from Eq. (3):

$\chi^{2}=\Sigma_{j=1}^{N} \frac{\left(I_{\infty, j}-H_{0, j} Q_{0} \Gamma(\delta+1) \beta_{j}^{-(\delta+1)}\right)}{\sigma_{\infty, j}^{2}}$

where the summation is carried out over $N$ active regions ( $j=1, . ., \mathrm{N})$, and $\sigma_{\infty, j}$ are measurement errors assigned to the adjusted intensities $I_{\infty, j}$, as explained later in Sect. 3 .

In general, a non-linear optimisation method could be used to derive values $Q_{\min }, \delta_{\min }$ that minimise $\chi^{2}$. In our case, however, since we only have two unknown parameters, we follow a simpler procedure which will also explicitly provide confidence limits on $\delta$. We create an array of values of $\delta$ from 0 to 3.0, with a step of 0.01 . For each of these values $\delta_{i}, i=$ $1, \ldots, K$, we analytically calculate $Q_{0, i}$ that minimises $\chi^{2}$ and calculate the corresponding minimum value of $\chi_{i}^{2}$. The smallest value among all $\chi_{i}^{2}, i=1, \ldots, K$, is the global $\chi^{2}$ minimum, denoted $\chi_{\min }^{2}$ and the corresponding $Q_{\min }, \delta_{\min }$ provide the solution to the minimisation problem.

To derive the confidence interval for $\delta_{\min }$, we use the method described by Avni (1976) and Cash (1976), and also illustrated by Fludra et al. (1989). Using their nomenclature, among the two parameters, $\delta$ is considered the "interesting" parameter, and $Q_{0}$ is the "uninteresting" parameter. A $1-p$ confidence interval for $\delta$ consists of all values of $\delta$ for which $\chi^{2}$, when minimised over $Q_{0}$ with $\delta$ fixed, is less than $\chi_{\min }^{2}+\Delta \chi^{2}$, where $\chi_{\min }^{2}$ is the global minimum of $\chi^{2}$ derived above. The critical value $\Delta \chi^{2}$ is derived from tables of the $\chi^{2}$ statistic with one degree of freedom and significance level $p$. For $90 \%$ confidence $(p=0.1), \Delta \chi^{2}=2.71$.

An alternative numerical approach is to minimise $\chi^{2}$ given by Eq. (3) (instead of Eq. (10)), where the observed intensity $I_{\mathrm{obs}}$ is calculated directly from Eq. (1) through numerical integration in the range $(a, b)$ instead of the range $(0, \infty)$. This direct treatment would allow us to compute $\delta$ for any shape of the histograms $H(\phi)$. However, the preceding discussion of the Laplace transform offers insights into the nature of this inverse problem, the uncertainties in defining the value of $\delta$, and also provides analytical formulae relating integrated active region intensities to the slope of the histograms of the magnetic flux density, or to the total magnetic flux and the average magnetic flux density (see the next section).

\section{Application to EUV spectra}

To illustrate this method, we use the EUV line of Fe XVI $360.8 \AA$ emitted at coronal temperatures with the emissivity peak at around $2.0 \times 10^{6} \mathrm{~K}$. Measurements of this line in many active regions were made by the Coronal Diagnostic Spectrometer on SOHO as part of the CDS synoptic observations. We analyse 26 active regions observed in years 19961998 as they crossed the solar central meridian. Only regions without sunspots have been selected for this study to simplify the determination of the maximum magnetic flux density and avoid the debate how much the sunspot penumbral and umbral magnetic fields contribute to the coronal EUV emission.

\subsection{Data analysis and implementation of the method}

Since the Fe XVI $360.8 \AA$ line is emitted at temperatures above $1.6 \times 10^{6} \mathrm{~K}$, this line is only seen in active regions and bright points and as such is used to define the active region area. An intensity threshold (above the level of instrumental noise) has been set at $200 \mathrm{erg} \mathrm{cm}^{-2} \mathrm{~s}^{-1} \mathrm{sr}^{-1}$ to separate the active region from the background. The area of all image pixels above this threshold is measured and the total Fe XVI line intensity in this area is calculated.

The histograms of the photospheric magnetic flux density for each active region have been obtained from the full-disk magnetograms recorded by the MDI instrument on SOHO with $2^{\prime \prime} \times 2^{\prime \prime}$ spatial pixels. The magnetogram area has been limited to a rectangle encompassing a projected area of the Fe XVI emission, with a $30^{\prime \prime}$ margin from the furthest points of the perimeter of the Fe XVI area. The histograms have typically two components: an exponential decay for stronger fields above $50 \mathrm{G}$, and an enhanced distribution for weaker fields below $50 \mathrm{G}$. A large part of the weaker fields come from the quiet area adjacent to the active region.

An exponential function (Eq. (4)) is fitted to histogram data in the range above $50 \mathrm{G}$ for each active region, to yield a pair of coefficients $H_{0, j}, \beta_{j}(1 \leq j \leq 26)$. The lower fitting limit of $50 \mathrm{G}$ is chosen to eliminate the quiet sun area from consideration. The upper limits of the magnetic flux density present in each active region differ from region to region, usually not exceeding $500 \mathrm{G}$. 
The first step in calculating $I_{\text {tot }}$ for an active region is to define the integration limits $a$ and $b$ in Eq. (1). The upper limit in Eq. (1) is determined by the value of $b=\phi_{\text {up }}$ for which the fitted exponential decreasing histogram reaches the value of one, i.e., for a perfect exponential shape of the histogram there would be no pixels with flux density greater than $\phi_{\text {up }}$.

To choose the lower limit $a$ in Eq. (1) we note that it is common to define the active region magnetic area by setting a higher threshold of the magnetic flux density, for example, $50 \mathrm{G}$ (e.g., Schrijver 1987). However, it is possible to consider that even the weakest fields inside the active region area might be capable of producing the EUV emission, perhaps through the interaction with the stronger fields or by being magnetically connected to stronger field areas. Therefore, we will consider a range of values for $a$ between 0 and $50 \mathrm{G}$, and observe how it affects the value of $\delta$.

With these finite limits $a$ and $b$, Eq. (1) describes how the observed line intensity $I_{\text {tot }}$ is produced, while Eq. (5) is an idealised mathematical formula defining intensity $I_{\infty}$ that would be produced if the magnetic flux density distribution were a perfect exponential in the whole range $(0, \infty)$. Thus, the Laplace inversion of Eq. (5) has to be coupled with finding the value of $I_{\infty}$. This can be done iteratively. In the first step, we assume

$I_{\infty}=I_{\mathrm{tot}}$

and derive a $\delta$ that minimises $\chi^{2}$ in Eq. (10). In the next iteration, we set

$I_{\infty}=I_{\text {tot }} \times \int_{0}^{\infty} \phi^{\delta} \exp (-\beta \phi) \mathrm{d} \phi / \int_{a}^{b} \phi^{\delta} \exp (-\beta \phi) \mathrm{d} \phi$

and fit a new value of $\delta$ that minimises $\chi^{2}$, then calculate a new $I_{\infty}$ with the above formula and so on until the change in $\delta$ becomes negligible after several iterations.

We also need to comment on the estimation of the errors $\sigma_{\infty, j}$ in Eq. (10). Since the line intensities are summed over the whole active region area, the statistical errors arising from the photon counting statistics are very small, less that $1 \%$. Therefore, any deviations between the observed intensities $I_{\text {tot }}$ and intensities predicted by Eq. (1) will be predominantly caused by the inaccuracies of the assumed model (Eq. (8)), for example, due to the assumption of constant $Q_{0}$ or other reasons discussed later in this section. We use these deviations to estimate the errors $\sigma_{\infty, j}$, by first setting $\sigma_{j}=1$, computing minimum $\chi^{2}$, and calculating an average relative error $\sigma_{\text {rel }}=\left|I_{\text {tot }, j}-I_{\text {pred }, j}\right| / I_{\text {tot }, j}$. In our case, $\sigma_{\text {rel }} \approx 0.3$. Subsequently, we assume that the relative error is the same in each active region, i.e., that $\sigma_{j}$ is proportional to the total intensity, $\sigma_{j}=\sigma_{\text {rel }} I_{\text {tot }}$. Errors $\sigma_{\infty, j}$ on the adjusted intensity $I_{\infty, j}$ are given by $\sigma_{\infty, j}=\sigma_{\text {rel }} I_{\infty, j}$.

Figure 2 shows $\chi^{2}$ as a function of $\delta$, obtained for $a=0 \mathrm{G}$. The minimum value of $\chi^{2}$ is reached for $\delta=1.3$. The $90 \%$ confidence interval is $(1.0,1.6)$. Figure 3 shows how the parameter $\delta$ and its $90 \%$ confidence interval varies as a function of $a$. As $a$ increases, the optimum $\delta$ decreases.

For future comparisons with the coronal heating models it is useful to establish the largest possible value of $\delta$. In our case

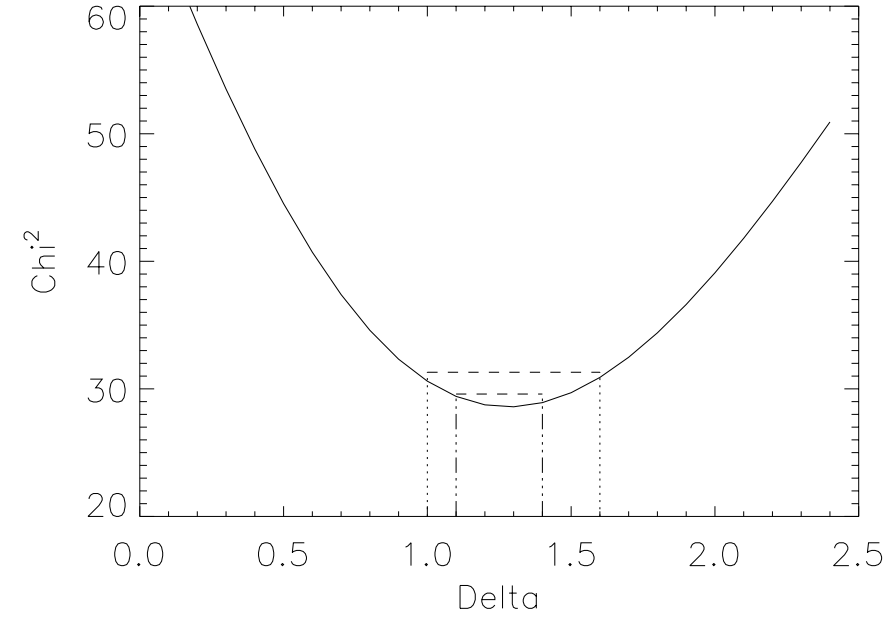

Fig. 2. A dependence of the goodness-of-fit criterion $\chi^{2}$ on the power index $\delta$, for the lower integration limit of $a=0 \mathrm{G}$. The minimum value of $\chi^{2}$ is reached for $\delta_{\min }=1.3$. Vertical dash-dot lines show a $67.8 \%$ confidence interval on $\delta_{\min }$, and vertical dotted lines show a $90 \%$ confidence interval.

this corresponds to $a=0$ for which we obtain that the intensity contribution from a magnetic pixel with an unsigned photospheric magnetic flux density $\phi$, to the total intensity of the Fe XVI $360.8 \AA$ line in each coronal loop is proportional to $\phi^{\delta}$, where $\delta=1.3$ and the $90 \%$ confidence interval is $1.0<\delta<1.6$. The total intensity of the Fe XVI $360.8 \AA$ line in each coronal loop is therefore proportional to $\Sigma \phi_{j}^{\delta}$, where $\phi_{j}$ are unsigned magnetic flux densities in all pixels located at its footpoints. The $90 \%$ confidence interval $(1.0,1.6)$ is rather large but does provide an upper limit on $\delta$. The ability to place an upper limit on $\delta$ is very useful in distinguishing among various heating models and will be explored in a future work.

Figure 4 shows the relationship between $\log \left(I_{\infty} / H_{0}\right)$ and $\log (\beta)$ based on Eq. (9). Intensities $I_{\infty}$ in Fig. 4 have been calculated using the observed Fe XVI intensities $I_{\text {tot }}$ according to the procedure described above. The continuous line represent the final best fit with $\delta=1.3$.

Some scatter of points around the fit to the distribution in Fig. 4 can be seen. There are several possible reasons for this scatter. Firstly, it may be that not all of the magnetic loops contain plasma emitting the Fe XVI $360.8 \AA$ line, and hence the proportion of Fe XVI-emitting loops may vary from region to region. Secondly, for those loops that have different magnetic flux densities at each footpoint, the actual dependence of the total line intensity on the flux density at both footpoints may be more complex than expressed by the relationship $I_{\text {loop }}=\Sigma I_{j}(\phi)$. Thirdly, the heating rate depends on the loop length which varies among individual loops in each active region; additionally, the distribution of loop lengths may also vary from region to region.

\subsection{Dependence of intensity on active region length scale}

Our analysis assumed a constant $Q_{0}$ over all active regions in Eq. (8). However, if $Q_{0}$ in truth depends on some averaged, 


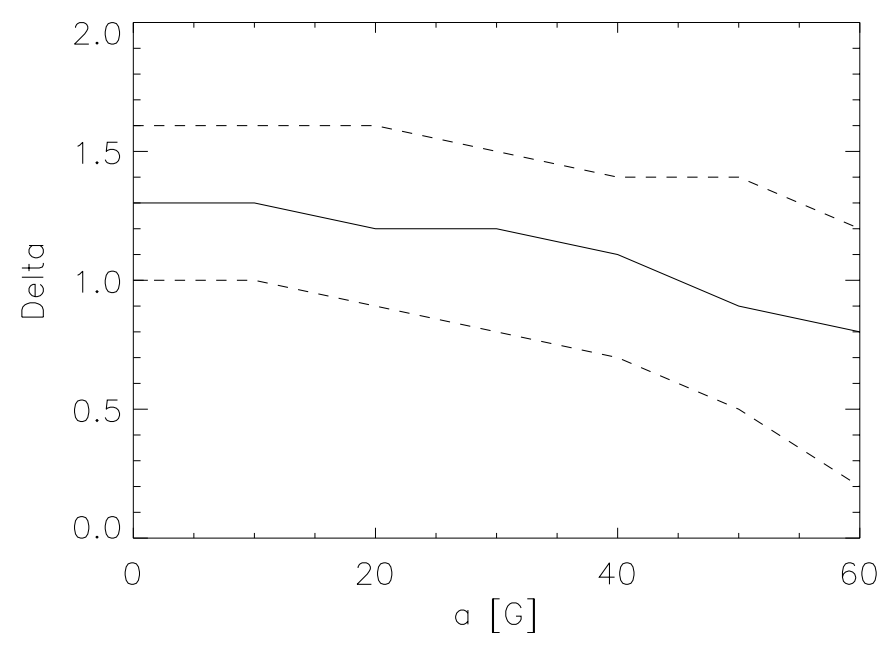

Fig. 3. A dependence of the power index $\delta$ on the lower integration limit (continuous line). Dashed lines show the $90 \%$ confidence interval.

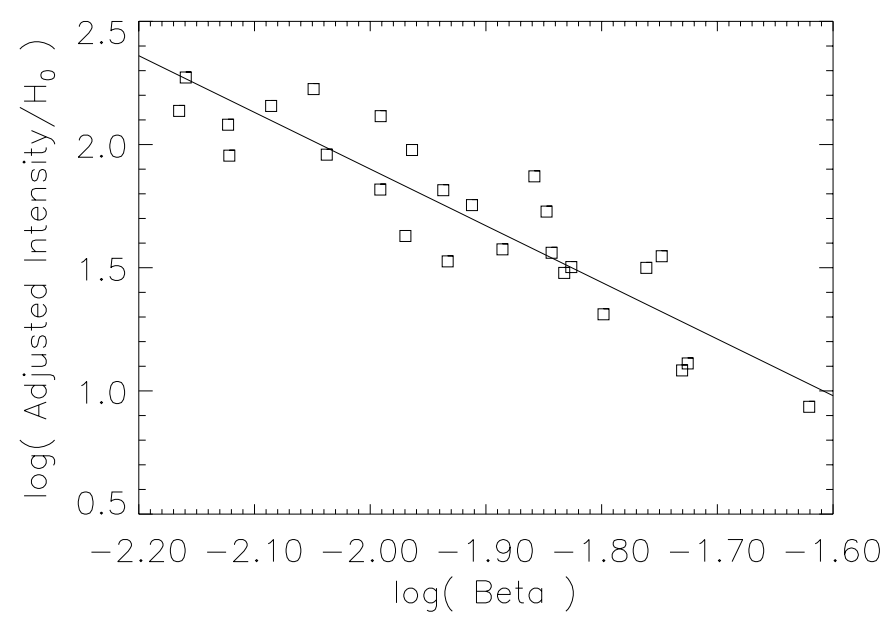

Fig. 4. A dependence of the quantity $I_{\infty} / H_{0}$ on the exponent of the magnetic field distribution (squares) for 26 active regions without sunspots. Continuous line is a linear fit with a slope of $-(\delta+1)=-2.3$ (see Eq. (9)).

ensemble measure of the loop size, $L_{\mathrm{av}}$, then deviations from our model are to be expected.

One way to test if a length scale dependence is present in the determination of $\delta$, would be to assume the following dependence of $Q_{0}$ on $L$ for each coronal loop: $Q_{0}=Q_{1} L^{\lambda}$, where the power $\lambda$ is the same for all loops. However, not knowing individual loop lengths, we can at best set one value of $Q_{0, j}$ for each region: $Q_{0, j}=Q_{\mathrm{av}, j} L_{\mathrm{av}, j}^{\lambda}$, where $L_{\mathrm{av}, j}$ is an average loop length for a given active region $j$. Then, if we knew the average loop length in each region, we could calculate $Q_{0, j}$ for each region from Eq. (9) and seek a correlation with $L_{\mathrm{av}, j}, j=1, \ldots, N$.

We have estimated $L_{\mathrm{av}}$ in two ways. The first estimate sets $L_{\mathrm{av}}$ to be the square root of the projected area of the coronal emission. The second estimate is generated in a more elaborate fashion, involving the following steps: (a) estimating the shape of the neutral line from the MDI magnetograms, (b) finding directions perpendicular to the neutral line at each point along the neutral line, and (c) measuring the extent of the projected Fe XVI emission along each of those perpendicular directions. An average value of the distribution of those "perpendicular" lengths is then calculated and assumed to represent an average loop length in a given active region. Subsequently, we have calculated the quantity $Q_{0, j}$ from Eq. (9) for each active region, using the optimal value of $\delta=1.3$. We then plotted the values of $Q_{0, j}$ against the average loop lengths, $L_{\mathrm{av}, j}$. We find a large scatter that gives only a weak dependence, $Q_{0} \propto L_{\mathrm{av}}^{0.3}$, with a $1 \sigma$ error of 0.13 on the power index.

As an alternative approach, we have substituted $Q_{0}$ in Eq. (9) by the function $Q_{0} L_{\mathrm{av}}^{\lambda}$, and varied $\lambda$ from -1 to 1 , each time repeating the analysis from Sect. (2) to derive the parameter $\delta$, and keeping record of minimum values of $\chi^{2}$. The global minimum of $\chi^{2}$ was obtained also for $\lambda=0.3$. Moreover, the optimal value of $\delta$ that minimises $\chi^{2}$ is always $\delta \approx 1.3$ for all values of $\lambda$ between 0 and 0.6 . Thus, we conclude that while there may be a weak dependence of the area-integrated line intensity on the average loop length, the determination of $\delta$ is essentially unaffected by the average loop length.

It may be that in some circumstances the value of $\delta$ determined by our method could be affected by the individual loop lengths. For example, one could propose a case in which the loop length has a special relationship with the magnetic flux density at its footpoints, $L \propto \phi^{v}$. In this particular case, when the individual loop intensity is proportional to $Q_{0} L^{\lambda} \phi^{\delta}$, the explicit dependence on $L$ would disappear and be absorbed into the power index over the magnetic flux density $\phi$, $I(\phi)=Q_{0} \phi^{\delta+v \lambda}$. In such case, a lack of dependence between $I_{\text {tot }}$ and $L_{\mathrm{av}}$ would not preclude the existence of the $I-L$ dependence for individual loops.

\section{Discussion}

Despite the deviations of our idealised model of active region emission from real active regions, the agreement between the fitted line and the data points in Fig. 4 is acceptable, confirming that the model $I_{\text {loop }} \propto \Sigma \phi_{j}^{\delta}$ is a reasonable approximation.

Our model given by Eq. (8) is equivalent to a generalised problem of the dependence of the integrated coronal emission on the moments of the magnetic flux density. The past research of Schrijver (1987) and Fisher et al. (1998) dealt with a small subset of this general problem. Three values of $\delta$ in Eq. (8) correspond to specific cases explored by Schrijver (1987) and Fisher et al. (1998): (1) $\delta=0$ is equivalent to calculating the total magnetic area; (2) $\delta=1.0$ is equivalent to calculating the total unsigned magnetic flux; and (3) $\delta=2.0$ is equivalent to the total of the magnetic flux density squared $\left(\Sigma \phi^{2} H(\phi)=\right.$ $\left.\int_{\text {area }} \phi^{2} \mathrm{~d} \phi\right)$.

Our finding that $\delta=1.0-1.3$ gives the best fit to the observed intensities of the Fe XVI line supports the conclusion of Fisher et al. (1998) that the X-ray luminosity correlates better with the total magnetic flux $\int \phi \mathrm{d} \phi$ than it correlates with $\int \phi^{2} \mathrm{~d} \phi$. Although Fisher et al. (1998) formulated the problem in terms of seeking a better correlation between only two possibilities, $\Sigma \phi H(\phi)$ and $\Sigma \phi^{2} H(\phi)$, and did not explore the intermediate values of $\delta$, nevertheless, serendipitously, the total magnetic flux appears to have been a good choice to explore in their 
analysis as it does correlate best with the Fe XVI intensity in our sample of active regions.

It can be shown that the proportionality of the total line intensity to the total magnetic flux follows naturally from our adopted model. Equation (9) can be expressed as

$I_{\infty}=Q_{0} \Gamma(\delta+1) B_{\mathrm{av}}^{\delta-1} \Phi$

because the total flux $\Phi=H_{0} \beta^{2}$ and the average magnetic flux density $B_{\text {av }}=1 / \beta$. Thus, for $\delta=1$, Eq. (11) predicts $I_{\infty} \propto$ $\Phi$. Even for $\delta \neq 1$, since $B_{\text {av }}$ varies only by a factor of two while $\Phi$ varies by a factor of 30 in our sample of 26 active regions, the intensity $I_{\infty}$ would still be proportional to $\Phi$ to a good approximation.

Figure 5 shows the dependence of the total Fe XVI intensity on the total magnetic flux. The continuous line represents a power law with a power index of $1.10 \pm 0.07$. This power index is close to the value of 1.2 found by Schrijver (1987) and Fisher et al. (1998) for the X-ray emission. As shown above, this global relationship is a manifestation of an underlying relationship between the intensity of individual loops and the magnetic flux density at their footpoints.

In contrast to previous studies which presented power laws relating the spatially-integrated line intensity and the total magnetic flux, this method offers a relationship between the intensity and the magnetic flux density at the footpoints for individual loops, assuming that this relationship is the same for all loops. This modelling assumption allows one to look at the distribution of magnetic flux elements in an active region, rather than their total. This approach treats the loops as an ensemble of similar objects rather than smearing them out completely as the studies of Schrijver (1987) and Fisher et al. (1998) do. In this regard, therefore this approach sits somewhere between the methods of Fisher et al. (1998), where the active region is reduced to two numbers (total X-ray flux and total unsigned magnetic field strength) and Aschwanden et al. (1999) who considers the properties of a number of individual loops.

In the context of this model, the emphasis is on the $I-\phi$ dependence, as the magnetic field is considered a primary factor in coronal heating mechanisms and is a well observed quantity at the photospheric level. The individual loop length enters as a parameter, but is very difficult to measure in our data. Thus, the question of how an individual loop intensity depends on the loop length is difficult to answer in the context of the approach outlined above as there is no direct length scale measurement available from observations. Instead, we can only estimate an average loop length for each active region (Sect. 3.2). We have tested to see if there is a dependence of the individual loop intensity on an average loop length but found that such average length dependence is not well established, as the value of $\lambda$ is small and its associated error is large (Sect. 3.2).

Certainly more successful is the establishment of a relationship between the coronal line intensity and the photospheric magnetic flux density in individual loops, which is the main goal of this paper. We have achieved an important step that prepares the way for deriving the dependence of the heating rate on the magnetic field. This next step will depend on the assumptions of loop equilibrium and loop models, and is a subject of further studies beyond the scope of the present paper.

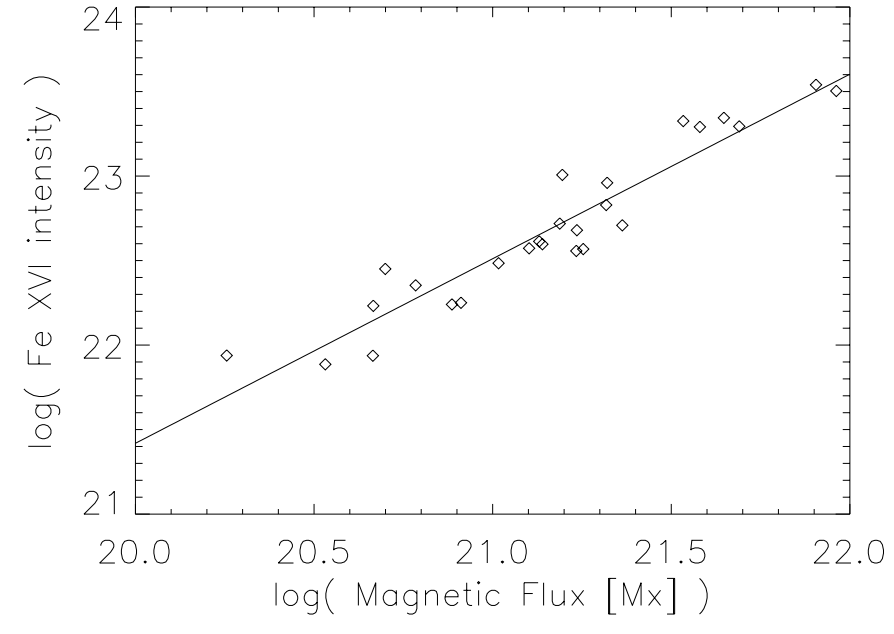

Fig. 5. The relationship between the spatially integrated Fe XVI intensity and the total magnetic flux for 26 active region plages (diamonds). The continuous line is a power law fit with a slope $1.10 \pm 0.07$.

Acknowledgements. This work was supported by the UK Particle Physics and Astronomy Research Council. SOHO is a project of international cooperation between ESA and NASA. J. Ireland acknowledges support from ESA Fellowship Programme and the European Solar Magnetometry Network.

\section{References}

Avni, Y. 1976, ApJ, 210, 642

Aschwanden, M. J., Newmark, J. S., Delaboudiniere, J.-P., et al. 1999, ApJ, 515, 842

Cash, W. 1976, A\&A, 52, 307

Craig, I. J. D., \& Brown, J. C. 1986, Inverse Problems in Astronomy (Hilger, Bristol)

Dere, K. P., Landi, E., Mason, H. E., Monsignori Fossi, B. C., \& Young, P. R. 1997, A\&AS, 125, 149

Fisher, G. H., Longcope, D. W., Metcalf, T. R., \& Pevtsov, A. A. 1998, ApJ, 508, 885

Fludra, A., \& Ireland, J. 2002, Proceedings of the 12th Cambridge Workshop on Cool Stars, Stellar Systems and the Sun, in press

Fludra, A., Lemen, J. R., Jakimiec, J., Bentley, R. D., \& Sylwester, J. 1989, ApJ, 344, 991

Fludra, A., \& Sylwester, J. 1986, Sol. Phys., 105, 323

Gurman, J. B., Withbroe, G. L., \& Harvey, J. W. 1974, Sol. Phys., 34, 105

Harrison, R. A., Sawyer, E. C., Carter, M. K., et al. 1995, Sol. Phys., 162,233

Mandrini, C. H., Demoulin, P., \& Klimchuk, J. A. 2000, ApJ, 530, 999

Martens, P. C. H., Kankelborg, C. C., \& Berger, T. E. 2000, ApJ, 537, 471

Porter, L. J., \& Klimchuk, J. A. 1995, ApJ, 454, 499

Rabin, D. 1992, ApJ, 391, 832

Rosner, R., Tucker, W. H., \& Vaiana, G. S. 1978, ApJ, 220, 643

Scherrer, P. H., Bogart, R. S., Bush, R. I., et al. 1995, Sol. Phys., 162, 129

Schrijver, C. J. 1987, A\&A, 180, 241

Schrijver, C. J., Title, A. M., Hagenaar, H. J., \& Shine, R. A. 1997, Sol. Phys., 175, 329

Schrijver, C. J., \& Aschwanden, M. J. 2002, ApJ, 566, 1147

Stenflo, J. O. 1973, Sol. Phys., 32, 41

Summers, H. P. 1993, JET Joint Undertaking Report, JET-IR(93)06 